\title{
Weak equilibrium in a spatial model
}

\author{
Manuel Abellanas • Ma Dolores López • \\ Javier Rodrigo • Isabel Lillo
}

\begin{abstract}
Spatial models of two-player competition in spaces with more than one dimension almost never have pure-strategy Nash equilibria, and the sludy of the equilibrium positions, if they exist, yields a disappointing result: the two players must choose the same position to achieve equilibrium. In this work, a discrete game is proposed in which the existence of Nash equilibria is studied using a geometric argument. This includes a definition of equilibrium which is weaker than the classical one to avoid the uniqueness of the equilibrium position. As a result, a "region of equilibrium" appears, which can be located by geometric methods. In this area, the players can move around in an "almost-equilibrium" situation and do not necessarily have to adopt the same position.
\end{abstract}

Keywords Nash equilibrium · Computational geometry - Game theory · Localion

\author{
M. Abellanas \\ Departamento de Matemática Aplicada de la Facultad de Informatica, Universidad Politécnica \\ de Madrid, Madrid, Spain

\section{D. López} \\ Departamento de Matemática Aplicada de la E.T.S.I. Caminos, Canales y Puerlos, \\ Universidad Politécnica de Madrid, Ciudad Universitaria s/n, 28040 Madrid, Spain \\ c-nail: marilo.lopez@upm.es \\ J. Rodrigo \\ Departamento de Matemática Aplicada, E.T.S. de Ingeniería, Universidad Pontificia Comillas \\ de Madrid, Madrid, Spain \\ 1. Lillo \\ IES Juan de la Cierva, Madrid, Spain
}




\section{Introduction}

The Nash equilibrium is studied in general models of competition. It was described for the first time by John Forbes Nash in his dissertation, Non-Cooperative Games (Nash 1951), as a way to obtain an optimum strategy for games with two or more players. Plott (1967), Kramer (1973), McKelvey (1976), and others have demonstrated that pure-strategy Nash equilibria generally do not exist when the competition takes place in a space with more than one dimension.

Various approaches have been presented in the literature in an attempt to resolve this situation: restricting the positions of the players, studying mixed-strategy Nash equilibria, or studying uncovered sets, among others (see, for example, Abellanas et al. 2006, 2010; Roemer 2001; McKelvey 1986).

In this work, a discrete model of competition between two players is presented, and the equilibrium positions are studied using a geometric analysis. This analysis involves geometric features such as convex hulls (de Berg et al. 1997). The uniqueness of the Nash equilibrium is stated, when this equilibrium exists: if there is Nash equilibrium in the game presented, then it is attained in a unique position. To avoid this situation a definition of equilibrium which is weaker than the classical one is proposed, to define a region from which the players should not deviate if they want to ensure that the other player cannot "greatly" improve his gain.

The game can be considered as a discrete version of the Voronoi game (Fekete and Meijer 2003), which is widely studied in computational geometry. It can be applied to some discrete competition models in different areas, as political competition.

The structure of the paper is as follows: Sect. 2 introduces the model. In Sect. 3 , necessary and sufficient conditions are presented for the Nash equilibrium positions to exist. Section 4 proposes a definition of equilibrium which is weaker than the classical one.

\section{The model}

This work generalizes the study of Nash equilibrium in a competitive game in which two players choose their positions in the plane to attract the largest possible number of points from $n$ fixed points on the plane.

Each player is considered to capture those points which lie closer to his position than to that of the other player. The perpendicular bisector of the players" locations thus partitions the plane into two regions. Each player wins the points in his own halfplane (Serra and Revelle 1994; Smid 1997; Aurenhammer and Klein 2000; Okabe et al. 2000). The goal of each player is to get the most points that can be achieved givent the behavior of the other player.

In formal terms, consider a strategic game $G=(N, X, \Pi)$, where $N=\{1,2\}$ is the set of two players, $X=\Re^{2} \times \Re^{2}$ is the strategy space, and $\Pi: X \rightarrow\{0, \ldots, n\} \times$ $\{0, \ldots, n\}$ is the payoff function. Moreover, the set of points each player wants to capture is denoted by $H=\left\{p_{1}, \ldots, p_{n}\right\} \subset R^{2}$. 
The description of the payoff function in the game presented here is given by:

$$
\begin{aligned}
& \begin{aligned}
\Pi^{1}\left(t_{1}, t_{2}\right) & =\text { number of points } p_{i} \text { such that } d\left(p_{i}, t_{1}\right) \leq d\left(p_{i}, t_{2}\right) \\
\Pi^{2}\left(t_{1}, t_{2}\right) & =\text { number of points } p_{i} \text { such that } d\left(p_{i}, t_{1}\right)>d\left(p_{i}, t_{2}\right) \\
& =n-\Pi^{1}\left(t_{1}, t_{2}\right)
\end{aligned} \\
& \text { if } t_{1} \neq t_{2}
\end{aligned}
$$

where $d\left(p_{i}, t\right)$ represents the Euclidean distance between the points $p_{i}, t$, and $\Pi^{i}, i=$ 1,2 represent the payoff of player $i$.

In the case where $t_{1}=t_{2}, \Pi^{1}\left(t_{1}, t_{2}\right)=\Pi^{2}\left(t_{1}, t_{2}\right)=\frac{n}{2}$ is defined.

Note that the game as presented here is a constant-sum game. Although the strategy space is infinite, the game can be considered as a discrete game in the sense that the image of $\Pi$ is a finite set. This is the main contribution of the paper compared to existing literature such us Rosenthal (1973) or Baron et al. (2004) where a finite strategy space is considered or for example Lindsey et al. (1995) where the payoff functions are not discrete.

\section{Equilibrium in the game}

\subsection{Existence of equilibrium}

In this section, necessary and sufficient conditions are developed for the existence of Nash equilibrium in the game defined in Sect. 2. Preliminary definitions are required.

Definition 1 A strategy profile $\left(t_{1}^{0}, t_{2}^{0}\right)$ is a Nash equilibrium if:

$$
\Pi^{1}\left(t_{1}, t_{2}^{0}\right) \leq \Pi^{1}\left(t_{1}^{0}, t_{2}^{0}\right), \quad \Pi^{2}\left(t_{1}^{0}, t_{2}\right) \leq \Pi^{2}\left(t_{1}^{0}, t_{2}^{0}\right) \quad \forall t_{1}, t_{2} \in \Re^{2}
$$

Definition 2 For fixed positive integer numbers $n, i$, consider the convex hulls of all possible selections of $\left[\frac{n}{2}\right]+i$ points from $H$. Define $C_{n, i}$ as the intersection of the convex hulls aforementioned ([] represents the floor function).

Proposition 1 There exist locations for a player in which the other player cannot obtain a payoff greater or equal than $\left[\frac{n}{2}\right]+1$ if and only if $C_{n, 1}$ is not empty. Any point in $C_{n, 1}$ will be one of those locations.

Proof $\Leftarrow$ If $C_{n, 1}$ is not empty, then any location of, say, player 1 at a point of $C_{n, 1}$ ensures that the other player cannot obtain any selection of $\left[\frac{n}{2}\right]+1$ points from $H$, because the position of player 1 is included in the convex hull of these $\left[\frac{n}{2}\right]+1$ points.

$\Rightarrow$ If $C_{n, 1}$ is empty, then for each position $t_{1}$ occupied by, say, player 1 , it is possible to find $\left[\frac{n}{2}\right]+1$ points of $H$ such that $t_{1}$ is not in their convex hull, and therefore there exist positions $t_{2}$ for player 2 that obtain these points $\left(t_{2}\right.$ could be the symmetric image of $t_{1}$ with respect to any straight line that separates the convex set and $t_{1}$, see Fig. 1). 
Fig. 1 Player 2 is located at the symnetrical inage of the position of player 1 with respect to a line that separates the convex set and the position of player 1

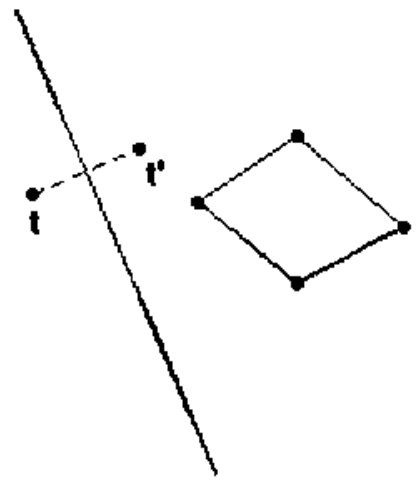

Now Proposition I can be used to find the Nash equilibrium positions in the proposed game.

Proposition 2 In the game presented here, Nash equilibrium positions exist if and only if $C_{n, 1}$ is not empty. In this case, the only Nash equilibrium positions will be any $\left(t_{1}, t_{2}\right)$ such that $t_{1}$ and $t_{2}$ are in this set.

Proof $\Leftarrow$ ) If $C_{n, 1}$ is not empty, then any position $\left(t_{1}, t_{2}\right)$ such that $t_{1}$ and $t_{2}$ belong to $C_{n, 1}$ is an equilibrium position.

If, say, the first player locates at $t_{1}$, then it is known (by Proposition 1 ) that player 2 cannot obtain more than $\frac{n}{2}$ points no matter where he locates, so $\Pi^{2}\left(t_{1}, t\right) \leq \frac{n}{2}$ for every $t$ in $R^{2}$. The same reasoning applies for the first player when the second one is at $t_{2}$.

On the other hand, in position $\left(t_{1}, t_{2}\right)$, each player obtains a payoff of $\frac{n}{2}$ points, because $\Pi^{1}\left(t_{1}, t_{2}\right) \leq \frac{n}{2}, \quad \Pi^{2}\left(t_{1}, t_{2}\right) \leq \frac{n}{2}$, and the gains are complementary. Therefore, $\Pi^{2}\left(t_{1}, t\right) \leq \frac{n}{2}=\Pi^{2}\left(t_{1}, t_{2}\right)$, and the same pattern exists for player 1 , so $\left(t_{1}, t_{2}\right)$ is a Nash equilibrium position.

These are the only equilibrium positions, for if $\left(t_{1}, t_{2}\right)$ is a Nash equilibrium position, then because $\Pi^{i}(t, t)=\frac{n}{2} \quad \forall t$ and a complementary payoff is assumed, $\Pi^{1}\left(t_{1}, t_{2}\right)=$ $\Pi^{2}\left(t_{1}, t_{2}\right)=\frac{n}{2}$. If, say, $t_{1}$ does not belong to $C_{n, 1}$, then there is a strategy for player 2 to obtain at least $\left[\frac{n}{2}\right]+1$ points of $H$ in a position $t$ (Proposition 1 ). Therefore, $\Pi^{2}\left(t_{1}, t\right) \geq\left[\frac{n}{2}\right]+1>\Pi^{2}\left(t_{1}, t_{2}\right)$, which is a contradiction because $\left(t_{1}, t_{2}\right)$ is a Nash equilibrium position.

$\Rightarrow$ ) If $C_{n, 1}$ is empty and there is a Nash equilibrium position $\left(t_{1}, t_{2}\right)$, then one of the payoff functions, say $\Pi^{2}$, satisfies the condition that $\Pi^{2}\left(t_{1}, t_{2}\right) \leq \frac{n}{2}$ because the gains are complementary. However, applying Proposition 1 , since $C_{n, 1}$ is empty, for position $t_{1}$ of player 1 , there exists a position $t$ for player 2 that obtains at least $\left[\frac{n}{2}\right]+1$ points of the set. Therefore, $\Pi^{2}\left(t_{1}, t\right) \geq\left[\frac{n}{2}\right]+1>\frac{n}{2} \geq \Pi^{2}\left(t_{1}, t_{2}\right)$, and player 2 can change his position profitably. This leads to a contradiction because $\left(t_{1}, t_{2}\right)$ is an equilibrium position. 


\subsection{Uniqueness of equilibrium}

The previous section established geometrically which are the Nash equilibrium positions for the game presented here, whether they exist, and where they are located. Now it will be determined when these positions are unique.

\subsubsection{Case of odd $n$}

Proposition 3 For odd $n, C_{n, 1}$ is empty or it is a non empty subset of $H$. Since the intersection of convex sets is a convex set, in this last case, $C_{n, 1}$ is a set consisting of a single point of $H$.

Proof If there is a point in $C_{n, 1}$ not belonging to $H$ and, say, player 1 is located at this point, then player 2 cannot gain $\left[\frac{n}{2}\right]+1$ points from the first player, by Proposition 1 , but for odd $n$, it is casy to sec that there is a stratcgy for a player to obtain $\left[\frac{n}{2}\right]+1$ points of $H$ if the other, say player 1, is situated in a position $t_{1}$ that is not a point of $H$ : just consider a line that separates $\left[\frac{n}{2}\right]+1$ points of $H$ of the rest of the points of $H$ and $t_{1}$ and locate player 2 in the symmetrical of $t_{1}$ with respect to this line. This yields a contradiction.

\subsubsection{Case of even $n$}

A similar result can be obtained for even $n$. A preliminary proposition is required.

Proposition 4 Let $p_{i}$ be a point of $H$ in the boundary of the convex hull of $H$. There exists a straight line that connects $p_{i}$ with another point of $H$ and that leaves the same number of points of the set in each half-plane.

Proof For any point of $H$ in the boundary of the convex hull, say $p_{1}$, it is possible to find a line containing this point that leaves the convex hull in a half-plane (de Berg et al. 1997). It can then be assumed without loss of generality that the convex hull is in the half-plane below the line (or on the right side of the line if it is a vertical line).

In this way, it is possible to assume that $p_{1}$ is the highest point of $H$ on the line and to arrange the other points of $H$ according to the angle of each with $p_{1}$. Because all the points of $H$ are in the same half-plane, it follows that the line that joins the middle point in the arrangement with $p_{1}$ leaves the same number of points on each side of the line (Fig. 2).

Fig. 2 Angular arrangement of points and selection of the point that. together with $p_{1}$. leaves half the points of $H$ on each side of the line

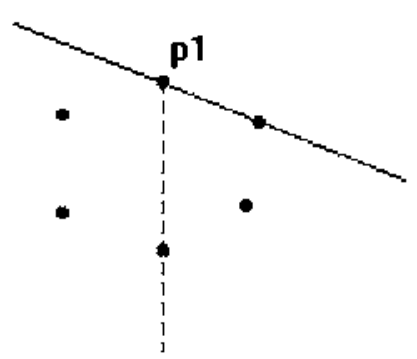


Remark. In the case of points not being in general position (there are more than two points in a same line) the line that joins the middle point in the arrangement with $p_{1}$ could contain other points of the set. In this case, the points of the set belonging to the segment joining $p_{1}$ and the middle point, are considered to be in the half-plane of the ones with less angle that the middle point, and the points of the set in the line not belonging to this segment, are considered to be in the other half-plane.

Proposition 5 For even $n$, if the $n$ points of $H$ are not collinear, then $C_{n, 1}$ is a point or the empty set.

Proof Select a point of $H$ on the boundary of the convex hull of the $n$ points. It is known by Proposition 4 that there is another point of the set such that the line connecting the two points leaves $\frac{n}{2}-1$ points on each side. Then the intersection of the convex hull of $\frac{n}{2}-1$ points on one side plus the two points on the line, and the convex hull of $\frac{n}{2}-1$ points on the other side plus the two points on the line, is a segment contained in the line. Because the points of $H$ are not collinear, there is a point on the boundary of the convex hull which is not on the line containing the segment, and by applying Proposition 4, it is possible to obtain another point of the set such that the line containing the two points leaves $\frac{n}{2}-1$ points on each side. Therefore, following the same reasoning as before, it is possible to find two convex hulls of $\frac{n}{2}+1$ points of the set whose intersection is another segment contained in the line, and then the intersection of the two segments is contained in the intersection of the two lines. It follows that the intersection of the two segments is contained in a set of one point. Therefore, the intersection of the four convex hulls is contained in a point, and therefore $C_{n, 1}$ is empty or a unique point.

Remark In the case of even $n$ and collinear points, there are an infinite number of equilibrium positions because $C_{n, 1}$ is the segment determined by the two intermediate points.

\subsubsection{General result}

Taking into account the results established in cases a) and b), it is possible to state the following general result:

Proposition $6 C_{n, 1}$ is a point or the empty set unless the points of $H$ are collinear with even $n$. Therefore, if there is Nash equilibrium in the game presented here, then this equilibrium is unique, with the two players choosing the same position $t$, unless the points are collinear with even $n$. Moreover, if $n$ is an odd number, then $t \in H$.

Remark It is possible to develop an algorithm that finds $C_{n, 1}$ with computational complexity of $\mathrm{O}($ nlogn $)$.

It can be seen that $C_{n, 1}$ is an empty set except for very special configurations of the points of $H$. Therefore, in the presented game, the equilibrium positions fail to exist except in singular cases. This result is analogous to the stated in Roemer (2001) for games similar to the presented one but with differentiable payoff functions. Nevertheless the discrete nature of the payoffs defined in this game, requires different techniques for the proofs that those used in Roemer (2001). 


\section{Weak equilibrium}

In this section, the discrete nature of the model is used to revise the definition of Nash equilibrium by means of:

Definition 3 A strategy profile $\left(t_{1}^{0}, t_{2}^{0}\right)$ is a weak equilibrium if:

$$
\Pi^{1}\left(t_{1}, t_{2}^{0}\right) \leq \Pi^{1}\left(t_{1}^{0}, t_{2}^{0}\right)+1, \quad \Pi^{2}\left(t_{1}^{0}, t_{2}\right) \leq \Pi^{2}\left(t_{1}^{0}, t_{2}^{0}\right)+1 \quad \forall t_{1}, t_{2} \in \Re^{2}
$$

This definition can be seen as a particular case of the $\varepsilon$-equilibrium studied by Monderer and Shapley (1996). Due the discrete nature of the game presented in the paper it makes sense taking $\varepsilon=1$. This is the motivation of Definition 3 .

A geometric analysis is developed here that extends the concepts presented in the previous section and underlies the search for the equilibrium positions, if they exist, according to this definition. For another generalization of the Nash equilibrium concept, see Aumann (1959).

\subsection{Existence of weak equilibrium}

Proposition 7 In a weak equilibrim position $\left(t_{1}^{0}, t_{2}^{0}\right)$, it is necessarily true that $\Pi^{1}\left(t_{1}^{0}, t_{2}^{0}\right) \geq \frac{n}{2}-1, \quad \Pi^{2}\left(t_{1}^{0}, t_{2}^{0}\right) \geq \frac{n}{2}-1$.

Proof Let $\left(t_{1}^{0}, t_{2}^{0}\right)$ be a weak equilibrium position. Assume that, say, $\Pi^{1}\left(t_{1}^{0}, t_{2}^{0}\right)<$ $\frac{n}{2}-1$. Then $\Pi^{1}\left(t_{2}^{0}, t_{2}^{0}\right)=\frac{n}{2}>\Pi^{1}\left(t_{1}^{0}, t_{2}^{0}\right)+1$, which is a contradiction because $\left(t_{1}^{0}, t_{2}^{0}\right)$ is a weak equilibrium position.

Remark As a consequence of this last proposition, because complementary payoffs are assumed, in a weak equilibrium position with even $n$, the payoffs must be $\frac{n}{2}-1, \frac{n}{2}+1$ or else $\frac{n}{2}$ for both. In a weak equilibrium position with odd $n$, the payoffs must be $\left[\frac{n}{2}\right]=\frac{n-1}{2},\left[\frac{n}{2}\right]+1=\frac{n+1}{2}$ or else both equal to $\frac{n}{2}$ (in this last case, the two players choose the same position).

The next step is to search for necessary and sufficient conditions for a position to be a weak equilibrium position.

Proposition 8 In the game presented here, there exist weak equilibrium positions if and only if $C_{n, 2}$ is not empty $(n>2)$.

Proof $\Leftarrow$ ) Let $t$ be a point belonging to $C_{n, 2}$. It will be shown that position $(t, t)$ is a weak equilibrium position.

$\Pi^{1}(t, t)=\Pi^{2}(t, t)=\frac{n}{2}$. If, say, $\Pi^{1}\left(t_{1}, t\right) \geq\left[\frac{n}{2}\right]+2$ for some position $t_{1}$ occupied by the first player, then there exists a straight line that separates at least $\left[\frac{n}{2}\right]+2$ points of $H$ from $t$, so $t$ does not belong to the convex hull of these $\left[\frac{H}{2}\right]+2$ points. This contradicts the initial assumption.

Remark The proof of the other implication of the proposition is analogous to that performed in the case studied in the previous section (Proposition 2). 
Now the weak equilibrium positions will be characterized according to the parity of $n$.

\section{1 .1 Case of odd $n$}

1. The weak equilibrium positions are $\left(t_{1}, t_{2}\right)$, with $\Pi^{1}\left(t_{1}, t_{2}\right)=\left[\frac{n}{2}\right], \quad \Pi^{2}\left(t_{1}, t_{2}\right)=$ $\left[\frac{n}{2}\right]+1, t_{1}$ belonging to $C_{n, 3}$, and $t_{2}$ in $C_{n, 2}$. In this situation, player 2 cannot earn $\left[\frac{n}{2}\right]+3$ points to increase by two his payoft in any position, and likewise player 1 cannot obtain $\left[\frac{n}{2}\right]+2$ points in $H$. These are the only possible weak equilibrium positions with these payoffs. If one of the players is not in the set under consideration, the other one can separate that player from at least $\left[\frac{n}{2}\right]+3$ or at least $\left[\frac{n}{2}\right]+2$ points respectively of the set by changing his position and increasing his gain by at least two units.

These positions make sense when $\left[\frac{n}{2}\right]+3 \leq n$, that is to say, $n \geq 5$.

2. The other weak equilibrium positions are $(t, t)$ with $t$ belonging to $C_{n, 2}(n>1)$.

There are no more weak equilibrium positions with other payoffs because they would not satisfy the necessary condition (Proposition 7).

\subsubsection{Case of even $n$}

The weak equilibrium positions will be:

1. Those in which one of the players has gain $\frac{n}{2}-1$ and is located in $C_{n, 3}$, and the other has gain $\frac{n}{2}+1$ and is located in $C_{n, 1}$, assuming that this last set is not empty and $n>4$. In these positions, neither of the players can increase his score by two by moving. These are the only possible weak equilibrium positions with these gains.

2. The other weak equilibrium positions are those where the two players each obtain half the payoff and are in $C_{n, 2}$.

\subsection{Examples}

For odd $n$, with $n>1$, every position $\left(t_{1}, t_{2}\right)$ such that $t_{1}, t_{2}$ are in $C_{n, 2}$ is a weak equilibrium position, because these positions with $t_{1} \neq t_{2}$ ensure that the payoffs of the players are $\left[\frac{n}{2}\right],\left[\frac{n}{2}\right]+1$. This is equivalent to case a), but these are not the only weak equilibrium positions.

For example, for $n=5$ and the set of points shown in Fig. 3, $C_{5,2}$ is the shaded polygon.

If $t_{1}, t_{2}$ are the positions labeled in Fig. 3, then $\Pi^{1}\left(t_{1}, t_{2}\right)=2, \quad \Pi^{2}\left(t_{1}, t_{2}\right)=3$.

Because $t_{2}$ belongs to $C_{5,2}$, the first player cannot change his position to win four points of $H$, so he cannot improve his payoff by two units. Likewise, because $t_{1}$ is inside the polygon defined by the points of $H$, the second player cannot win five points in any position, and therefore they are in a weak equilibrium position, but $t_{1}$ does not belong to $C_{5,2}$. 
Fig. 3 Points in a weak equilibrium position for odd $n$

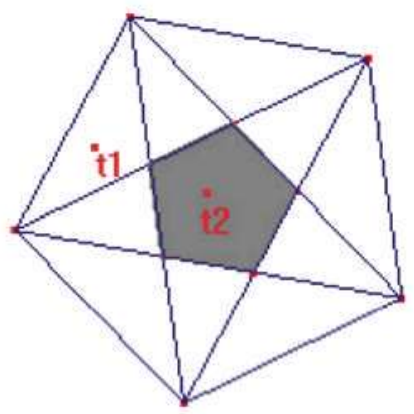

Fig. 4 Points in a weak equilibrium position for even $n$

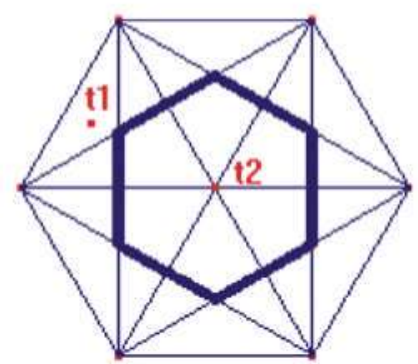

Note that in this set there is no Nash equilibrium position because $C_{5,1}$ is empty (Proposition 3). Therefore, the search for weak equilibrium in this example appears to be interesting.

For even $n, n>2$, it should be noted that positions $\left(t_{1}, t_{2}\right)$ with $t_{1}, t_{2}$ in $C_{n, 2}$ are not necessarily weak equilibrium positions because these positions do not ensure the payoffs stated in case b). There are also, as in the previous case, weak equilibrium positions in which one of the parties is not in $C_{n, 2}$. For example, for $n=6$, in the situation shown in Fig. 4, the gains are $\Pi^{1}\left(t_{1}, t_{2}\right)=2, \quad \Pi^{2}\left(t_{1}, t_{2}\right)=4, t_{2}$ is in $C_{6,1}, t_{1}$ is in $C_{6,3}$, and therefore $\left(t_{1}, t_{2}\right)$ is a weak equilibrium position as in case b), but $t_{1}$ is not in $C_{6,2}$.

This example yields a unique Nash equilibrium position, $\left(t_{2}, t_{2}\right)$, meaning that the two players choose the same position, but it has an infinite number of weak equilibrium positions.

\subsection{Generalization}

In this section a generalization of the weak equilibrium concept is introduced by means of the following definition:

Definition 4 A strategy profile $\left(t_{1}^{0}, t_{2}^{0}\right)$ is a $k$-equilibrium if:

$$
\Pi^{1}\left(t_{1}, t_{2}^{0}\right) \leq \Pi^{1}\left(t_{1}^{0}, t_{2}^{0}\right)+k, \quad \Pi^{2}\left(t_{1}^{0}, t_{2}\right) \leq \Pi^{2}\left(t_{1}^{0}, t_{2}^{0}\right)+k \quad \forall t_{1}, t_{2} \in \Re^{2}
$$

( $k$ is an integer number with $0 \leq k \leq\left[\frac{n}{2}\right]$ if $n$ is an odd number, $0 \leq k \leq \frac{n}{2}-1$ if $n$ is an even number). 
Remark 0-equilibrium is equivalent to Nash equilibrium and 1-equilibrium is equivalent to weak equilibrium.

The results obtained in the previous sections can be easily translated to $k$-equilibrium definition as follows:

Proposition 9 In a $k$-equilibrium position $\left(t_{1}^{0}, t_{2}^{0}\right)$, it is necessarily true that $\Pi^{1}\left(t_{1}^{0}, t_{2}^{0}\right) \geq \frac{n}{2}-k, \quad \Pi^{2}\left(t_{1}^{0}, t_{2}^{0}\right) \geq \frac{n}{2}-k$.

Proposition 10 In the game presented here, there exist $k$-equilibrium positions if and only if $C_{n,(k+1)}$ is not empty.

It can be seen that greater values of $k$ provide bigger $k$-equilibrium regions but this situation carries less degree of stability.

\subsection{Political application}

The discrete game and the generalization of the concept of equilibrium showed in this paper can be applied to multiple doctrines, especially those related to problems of location.

As an example it can be given an interpretation in terms of political competition in the following way:

The two players are two political parties which compete in an election. The positions they choose in the plane are the policies they adopt in two certain important items in the electoral process. The set of points each player wants to capture are the voters in the election.

We have proved that when Nash equilibrium positions exist in the game presented, these are unique and they are reached when the two players choose the same strategy. That is to say in terms of this example, the two parties will propose, in essence, a similar political program to ofter to their voters. This is not a very realistic situation. The consideration of a generalization of the equilibrium is then a contribution for avoiding this problem because it permits more political options to the parties which assure them positions very much close to stability.

A version of this layout, supposing that the set of voters is a continuum, can be seen in Roemer (2001).

\section{Conclusions}

In equilibrium analysis of most competitive multidimensional games with two players, it has been found that such positions do not exist except for singular cases, and therefore there are no positions for the players that guarantee that the other player cannot increase his gain by moving. A discrete two-dimensional competition model has been proposed and analyzed using geometric strategies that find the Nash equilibrium positions if they exist and ensure their uniqueness. In spite of this, Nash equilibrium in the majority of the situations studied has been found not to exist. To resolve this situation, a weakened definition of equilibrium has been presented, which ensures 
for each player that the other cannot improve his payoff by more than one unit if he changes his position. This new definition of equilibrium can be useful in cases which have no Nash equilibrium.

Indeed, the weak equilibrium positions as defined in this research are usually regions of the plane, so they yield infinite possibilities for the players to move, in contrast to the single position available to the players in a Nash equilibrium.

The study of the existence and locations of Nash equilibrium and weak equilibrium positions has here been expanded in scope by applying techniques from computational geometry such as the intersection of convex hulls, which can be used because of the discrete nature of the game.

\section{References}

Abellanas M, Lillo I, López M. Rodrigo J (2006) Electoral strategies in a dynanical democratic system. Geometric models. Eur J Oper Res 175:870-878

Abellanas M, López MD, Rodrigo J (2010) Searching for eguilibrium positions in a game of political competition with restrictions. Eur J Oper Res 201:892-896. doi:10.1016/j.ejor.2009.04.002

Aumann RJ (1959) Acceptable points in general cooperative n-person games. Contributions to the Theory of Games IV. Ann Math Stud 40:287-324

Aurenhammer R, Klein R (2000) Voronoi diagrams. In: Sack J-R, Urrutia J (eds) Handbook of computational geometry. Elsevier Science Publishers. Amsterdan

Baron R, Durie J, Haller H. Solal P (2004) Finding a Nash equilibrium in spatial games is an NP-complete problem. Econ Theory 23:445-454

de Berg M, van Kreveld M. Overmars M. Schwarzkopf O (1997) Computational geometry-alyorithms and applications. 2nd edn. Springer. New York

Fekete S, Meijer H (2003) The one-round Voronoi game replayed. Workshop on algorithms and data structures. Springer Lect Notes Comput Sci 2748:150-161

Kramer GH (1973) On a class of equilibrium conditions for majority rule. Econonetrica 42:285-297

Lindsey J, Pratt J. Zeckhauser R (1995) Equilibrium with agglomeration economies. Reg Sci Urban Econ 25:249-260

McKelvey RD (1976) Intransitivities in multidimensional voting models and implications for agenda control. J Econ Theory 12:472-482

McKelvey RD (1986) Covering. dominance. and the institution-free properties of social choice. Am J Political Sci 30:283-314

Monderer D, Shapley LS (1996) Potential games. Games Econ Behav 14:124-143

Nash J (1951) Non-cooperative games. Ann Math 54:286-295

Okabe A. Boots B. Sugihara K. Chiu S (2000) Spatial tessellations concepts and applications of voronoi diagrams. Wiley. Chichester

Plott CR (1967) A notion of equilibrium and its possibility under majority rule. Am Econ Rev 57:787-806

Roemer J (2001) Political competition. Harvard University Press, London

Rosenthal RW (1973) A class of games possessing pure-strategy Nash equilibria. Int J Game Theory 2(1):65-67

Serra D. Revelle C (1994) Market capture by two competitors: The preemptive location problem. J Reg Sci 34(4):549-561

Smid M (1997) Closest point problems in computational geometry. In: Sack J-R. Utrutia J (eds) Handbook on computational geometry. Elsevier Science, Ansterdam 